\title{
Separability preserving Dirac reductions of Poisson pencils on Riemannian manifolds
}

\author{
Maciej Błaszak* \\ Institute of Physics, A. Mickiewicz University \\ Umultowska 85, 61-614 Poznań, Poland \\ Krzysztof Marciniak* ${ }^{\ddagger}$ \\ Department of Science and Technology \\ Campus Norrköping, Linköping University \\ 601-74 Norrköping, Sweden
}

November 11, 2018

\begin{abstract}
Dirac deformation of Poisson operators of arbitrary rank is considered. The question when Dirac reduction does not destroy linear Poisson pencils is studied. A class of separability preserving Dirac reductions in the corresponding quasi-bi-Hamiltonian systems of Benenti type is discussed. Two examples of such reductions are given. This paper will appear in J. Phys. A: Math. Gen.
\end{abstract}

AMS 2000 Subject Classification: 70H45,70H06,70H20,53D17,70G45

\section{Introduction}

Recently a new (quasi)-bi-Hamiltonian separability theory of Liouville integrable finite dimensional systems was constructed [1-[15] based on general properties of Poisson pencils on manifolds. A natural further step within this theory is to investigate admissible integrable and separable reductions of these integrable / separable systems onto appropriate submanifolds. The most natural approach seems to be the one based on the Dirac theory of constrained dynamics [16], [17]. The presented paper contains only some special cases of such reductions, but even in these cases the problem is far from being trivial. The difficulties we met during our research inclined us to reconsider the Dirac formalism from the point

\footnotetext{
*Partially supported by Swedish Research Council Grant No. 629-2002-6681

${ }^{\dagger}$ Partially supported by KBN grant No. 5P03B 00420

${ }^{\ddagger}$ On leave of absence from Department of Physics, A. Mickiewicz University, Poznań, Poland.
} 
of view of Poisson bivectors rather then from the point of view of constrained dynamics.

The paper is organized as follows. In this introductory part we remind basic concepts of Poisson geometry and of (quasi-)bi-Hamiltonian systems. In Section 2 we formulate the theory of Dirac reductions of Poisson brackets in terms of Poisson bivectors. Our construction is more general than usually met in literature since we consider reduction procedure on the whole foliation of submanifolds. Here we also explain that our approach indicates that Dirac classification of constraints onto those of "first" and of "second class" requires further discussion as our further results show. In Section 3 we review the recent results on separability theory of quasi-bi-Hamiltonian systems of Benenti type. In section 4 we perform Dirac reduction of Poisson pencil and corresponding quasi-bi-Hamiltonian chain of Benenti type onto a particularly chosen submanifold. Chosen constraints preserve the Liouville integrability as well as the coordinates of separation of considered system. The main obstacle of such a choice is that these constraints are nonexpressible in natural (original) coordinates. Hence, in Section 5 we modify constraints introduced in the previous section and obtain an equivalent reduction, that is expressible directly in original coordinates. Finally, in Section 6 we illustrate the results by two nontrivial examples of constrained separable dynamics.

Let us first remind few basic facts from Poisson geometry. Given a manifold $\mathcal{M}$, a Poisson operator $\pi$ on $\mathcal{M}$ is a mapping $\pi: T^{*} \mathcal{M} \rightarrow T \mathcal{M}$ that is fibrepreserving (i.e. $\left.\pi\right|_{T_{x}^{*} \mathcal{M}}: T_{x}^{*} \mathcal{M} \rightarrow T_{x} \mathcal{M}$ for any $x \in \mathcal{M}$ ) and such that the induced bracket on the space $C^{\infty}(\mathcal{M})$ of all smooth real-valued functions on $\mathcal{M}$

$$
\{., .\}_{\pi}: C^{\infty}(\mathcal{M}) \times C^{\infty}(\mathcal{M}) \rightarrow C^{\infty}(\mathcal{M}) \quad, \quad\{F, G\}_{\pi} \stackrel{\text { def }}{=}\langle d F, \pi d G\rangle
$$

(where $\langle.,$.$\rangle is the dual map between T \mathcal{M}$ and $T^{*} \mathcal{M}$ ) is skew-symmetric and satisfies Jacobi identity (the bracket (10) always satisfies the Leibniz rule $\{F, G H\}_{\pi}=$ $\left.G\{F, H\}_{\pi}+H\{F, G\}_{\pi}\right)$. Throughout the whole paper the symbol $d$ will denote the operator of exterior derivative. The operator $\pi$ can always be interpreted as a bivector, $\pi \in \Lambda^{2}(\mathcal{M})$ and in a given coordinate system $\left(x^{1}, \ldots, x^{m}\right)$ on $\mathcal{M}$ we have

$$
\pi=\sum_{i<j}^{m} \pi^{i j} \frac{\partial}{\partial x_{i}} \wedge \frac{\partial}{\partial x_{j}} .
$$

A function $C: \mathcal{M} \rightarrow \mathbb{R}$ is called Casimir function of the Poisson operator $\pi$ if for arbitrary function $F: \mathcal{M} \rightarrow \mathbb{R}$ we have $\{F, C\}_{\pi}=0$ (or, equivalently, if $\pi d C=0)$. A linear combination $\pi_{\xi}=\pi_{1}-\xi \pi_{0}(\xi \in \mathbb{R})$ of two Poisson operators $\pi_{0}$ and $\pi_{1}$ is called Poisson pencil if the operator $\pi_{\xi}$ is Poisson for any value of the parameter $\xi$. In this case we say that $\pi_{0}$ and $\pi_{1}$ are compatible. Given a Poisson pencil $\pi_{\xi}=\pi_{1}-\xi \pi_{0}$ we can often construct a sequence of vector fields $Y_{i}$ on $\mathcal{M}$ that have a twofold Hamiltonian form (a so called bi-Hamiltonian chain)

$$
Y_{i}=\pi_{1} d h_{i}=\pi_{0} d h_{i+1}
$$

where $h_{i}: \mathcal{M} \rightarrow \mathbb{R}$ are called Hamiltonians of the chain (2) and where $i$ is 
some discrete index. This sequence of vector fields may or may not truncate (depending on existence of Casimir functions). In the case when the Poisson pencil $\pi_{\xi}$ is degenerated but projectable into a symplectic leaf $\mathcal{N}$ (of dimension $2 n$ ) of $\pi_{0}$ the bi-Hamiltonian chain (2) on $\mathcal{M}$ turns into a so called quasi-biHamiltonian chain on $\mathcal{N}$ of the form

$$
\theta_{1} d H_{i}=\theta_{0} d H_{i+1}+\sum_{\substack{j=1 \\ j \neq i+1}}^{n} \alpha_{i j} \theta_{0} d H_{j}, \quad i=1, \ldots, n, \quad H_{n+1} \equiv 0,
$$

where $\theta_{i}$ are projections of $\pi_{i}$ onto $\mathcal{N}$, the functions $H_{j}$ are restrictions of $h_{j}$ to $\mathcal{N}: H_{j}=\left.h_{j}\right|_{\mathcal{N}}$, and $\alpha_{i j}$ are some multipliers (real functions). And vice versa: having a quasi-bi-Hamiltonian chain (3) on the manifold $\mathcal{N}$ one can lift it to a bi-Hamiltonian chain (2) on the extended manifold $\mathcal{M}$. (Quasi-)biHamiltonian chains (called also (quasi-)bi-Hamiltonian systems) possess very interesting differential-algebraic properties and are one of key notions in the theory of integrable systems, due to the fact that in many cases the systems (2) and (3) are Liouville integrable [18. Recently, much effort has been spent in order to exploit the procedure of solving these systems by the method of separation of variables [2]-15]. In this article we will mainly work with the quasibi-Hamiltonian chains (3) rather than bi-Hamiltonian ones, since the pencil $\theta_{\xi}=\theta_{1}-\xi \theta_{0}$ is always non-degenerated.

\section{Dirac reduction of Poisson bivectors}

We begin by considering the Dirac reduction procedure in a more general setting that is usually met in literature. Let $\pi$ be a Poisson bivector, in general degenerated on some manifold $\mathcal{M}$. Let $\mathcal{S}=\mathcal{F}_{0}$ be a submanifold in a foliation $\mathcal{F}$ of the manifold $\mathcal{M}$ defined by $m$ functionally independent functions (constraints) $\varphi_{i}: \mathcal{M} \rightarrow \mathbb{R}, i=1, \ldots, m$.

$$
\mathcal{F}_{s}=\left\{x \in M: \varphi_{i}(x)=s_{i}, i=1, \ldots, m\right\}
$$

Thus, $\mathcal{S}$ is a submanifold of codimension $m$ in $\mathcal{M}$. Moreover, let $Z_{i}, i=1, \ldots, m$ be some vector fields transversal to $\mathcal{F}_{s}$, spanning a regular distribution $\mathcal{Z}$ in $\mathcal{M}$ of constant dimension $m$ (that is a smooth collection of $m$-dimensional subspaces $\mathcal{Z}_{x} \subset T_{x} \mathcal{M}$ at every point $x$ in $\mathcal{M}$ ). The word 'transversal' means here that no vector field $Z_{i}$ is at any point tangent to the submanifold $\mathcal{F}_{s}$ passing through this point. Hence, the tangent bundle $T \mathcal{M}$ splits into a direct sum

$$
T \mathcal{M}=T \mathcal{F} \oplus \mathcal{Z}
$$

(which means that at any point $x$ in $\mathcal{M}$ we have $T_{x} \mathcal{M}=T_{x} \mathcal{F}_{s} \oplus \mathcal{Z}_{x}$ with $s$ such that $x \in \mathcal{F}_{s}$ ) and so does its dual

$$
T^{*} \mathcal{M}=T^{*} \mathcal{F} \oplus \mathcal{Z}^{*}
$$


where $T^{*} \mathcal{F}$ is the annihilator of $\mathcal{Z}$ and $\mathcal{Z}^{*}$ is the annihilator of $T \mathcal{F}$. That means that if $\alpha$ is a one form in $T^{*} \mathcal{F}$ then $\alpha\left(Z_{i}\right)=0$ for all $i=1, \ldots, m$ and if $\beta$ is a one-form in $\mathcal{Z}^{*}$ then $\beta$ vanishes on all vector fields tangent to $\mathcal{F}_{s}$. Moreover, we assume that the vector fields $Z_{i}$ which span $\mathcal{Z}$ are chosen in such a way that $d \varphi_{i}, i=1, \ldots, m$ is a basis in $\mathcal{Z}^{*}$ that is dual to the basis $Z_{i}$ of the distribution $\mathcal{Z}$

$$
\left\langle d \varphi_{i}, Z_{j}\right\rangle=Z_{j}\left(\varphi_{i}\right)=\delta_{i j}
$$

(this is no restriction since for any distribution $\mathcal{Z}$ transversal to $\mathcal{F}_{s}$ we can choose its basis so that (4) is satisfied). Finally, let us define $m$ vector fields $X_{i}$ on $\mathcal{M}$ and $m^{2}$ functions $\varphi_{i j}: \mathcal{M} \rightarrow \mathbb{R}$ on $\mathcal{M}$ through

$$
X_{i}=\pi\left(d \varphi_{i}\right), \quad \varphi_{i j}=\left\{\varphi_{i}, \varphi_{j}\right\}_{\pi}=\left\langle d \varphi_{i}, \pi d \varphi_{j}\right\rangle=X_{j}\left(\varphi_{i}\right) .
$$

The functions $\varphi_{i j}$ define an $m$-dimensional skew-symmetric matrix $\varphi=\left(\varphi_{i j}\right)$, $i, j=1, \ldots m$. It can be easily shown that

$$
\left[X_{j}, X_{i}\right]=X_{\left\{\varphi_{i}, \varphi_{j}\right\}_{\pi}}=\pi d\left\{\varphi_{i}, \varphi_{j}\right\}_{\pi}=\pi d \varphi_{i j}
$$

where $\{., .\}_{\pi}$ is a Poisson bracket defined by our Poisson bivector $\pi$ and $[X, Y]=$ $L_{X} Y=X(Y)-Y(X)$ is the Lie bracket (commutator) of the vector fields $X, Y$.

A very special choice of our transversal vector fields $Z_{i}$ originates by taking linear combinations of fields $X_{j}$ with coefficients being the entries of the matrix $\varphi^{-1}$.

$$
Z_{i}=\sum_{j=1}^{m}\left(\varphi^{-1}\right)_{j i} X_{j} \quad i=1, \ldots, m .
$$

Since the constraint functions $\varphi_{i}$ are functionally independent, the vector fields $Z_{i}$ in (77) will indeed be transversal to the foliation $\mathcal{F}$. Moreover, they will automatically satisfy the orthogonality condition (4) as $Z_{i}\left(\varphi_{j}\right)=\sum_{k=1}^{m}\left(\varphi^{-1}\right)_{k i} X_{k}\left(\varphi_{j}\right)$ $=\sum_{k=1}^{m}\left(\varphi^{-1}\right)_{k i} \varphi_{j k}=\delta_{i j}$.

Let us now consider the following deformation (modification) of the bivector $\pi$ :

$$
\pi_{D}=\pi-\frac{1}{2} \sum_{i=1}^{m} X_{i} \wedge Z_{i},
$$

where $\wedge$ denotes the wedge product in the algebra of multivectors. This new bi-vector $\pi_{D}$ can be properly restricted to $\mathcal{F}_{s}$ for any $s \in \mathbb{R}^{m}$ (and thus also to $\mathcal{S}=\mathcal{F}_{0}$ ), since the image of $\pi_{D}$ considered on a given leaf $\mathcal{F}_{s}$ of the foliation $\mathcal{F}$ lies in $T \mathcal{F}_{s}$ for all $s$. This is the content of the following theorem.

Theorem 1 Suppose $x \in \mathcal{F}_{s}$. Then for any $\alpha \in T_{x}^{*} \mathcal{M}$ the vector $\pi_{D}(\alpha)$ is tangent to $\mathcal{F}_{s}$ i.e. $\pi_{D}\left(T_{x}^{*} \mathcal{M}\right) \subset T_{x} \mathcal{F}_{s}$.

Proof. We will show, that $\pi_{D}\left(d \varphi_{k}\right)=0, k=1, \ldots, m$, since it means that the constraints $\varphi_{i}, i=1, \ldots, m$ are Casimirs of $\pi_{D}$ (and so are then $\varphi_{i}-s_{i}$ ) which obviously implies the thesis of the theorem. Using the definition (8) of 
$\pi_{D}$, the obvious fact that $\left(X_{i} \wedge Z_{i}\right) d \varphi_{k}=\left(X_{i} \otimes Z_{i}\right) d \varphi_{k}-\left(Z_{i} \otimes X_{i}\right) d \varphi_{k}=$ $\left\langle d \varphi_{k}, Z_{i}\right\rangle X_{i}-\left\langle d \varphi_{k}, X_{i}\right\rangle Z_{i}=Z_{i}\left(\varphi_{k}\right) X_{i}-X_{i}\left(\varphi_{k}\right) Z_{i}$ we have

$$
\begin{aligned}
\pi_{D}\left(d \varphi_{k}\right) & =\pi\left(d \varphi_{k}\right)-\frac{1}{2} \sum_{i} Z_{i}\left(\varphi_{k}\right) X_{i}+\frac{1}{2} \sum_{i} X_{i}\left(\varphi_{k}\right) Z_{i} \\
& =X_{k}-\frac{1}{2} \sum_{i} \delta_{i k} X_{i}+\frac{1}{2} \sum_{i, j} \varphi_{k i}\left(\varphi^{-1}\right)_{j i} X_{j} \\
& =X_{k}-\frac{1}{2} X_{k}-\frac{1}{2} \sum_{i} \delta_{k i} X_{i}=0
\end{aligned}
$$

due to the fact that $\varphi_{i j}=-\varphi_{j i}$.

Theorem 2 The bivector $\pi_{D}$ in (8) with $Z_{i}$ as in (7) satisfies the Jacobi identity.

Proof. It is easy to check that our operator $\pi_{D}$ defines the following bracket on $\mathcal{M}$

$$
\{F, G\}_{\pi_{D}}=\{F, G\}_{\pi}-\sum_{i, j=1}^{m}\left\{F, \varphi_{i}\right\}_{\pi}\left(\varphi^{-1}\right)_{i j}\left\{\varphi_{j}, G\right\}_{\pi},
$$

(where $F, G: \mathcal{M} \rightarrow \mathbb{R}$ are two arbitrary functions on $\mathcal{M}$ ) which is just the well known Dirac deformation [16] of the bracket $\{., .\}_{\pi}$ associated with $\pi$, and as was shown by Dirac [17] it satisfies the Jacobi identity.

Remark 3 If $C: \mathcal{M} \rightarrow \mathbb{R}$ is a Casimir function of $\pi$, then it is also a Casimir function of $\pi_{D}$, since in this case (2) yields

$$
\{F, C\}_{\pi_{D}}=\{F, C\}_{\pi}-\sum_{i, j=1}^{m}\left\{F, \varphi_{i}\right\}_{\pi}\left(\varphi^{-1}\right)_{i j}\left\{\varphi_{j}, C\right\}_{\pi}=0-0=0 .
$$

We also know from Theorem [1 that the constraints $\varphi_{i}$ are Casimirs of the deformed operator $\pi_{D}$. Thus, we can informally state that Dirac deformation preserves all the old Casimir functions and introduces new Casimirs $\varphi_{i}$.

It is now possible to restrict our Poisson operator $\pi_{D}$ (or our Poisson bracket $\{., .\}_{\pi_{D}}$ ) to a Poisson operator (bracket) on the submanifold $\mathcal{S}$ (i.e. a symplectic leaf $\varphi_{1}=\ldots=\varphi_{m}=0$ of $\pi_{D}$ ) in a standard way (reduction to a symplectic leaf). Namely, for arbitrary functions $f, g: \mathcal{S} \rightarrow R$ one defines the reduced Dirac bracket $\{f, g\}_{R}$ on $\mathcal{S}$ (with the corresponding Poisson operator $\pi_{R}$ ) as the restriction of the bracket of arbitrary prolongations of $f$ and $g$ to $\mathcal{M}$, i.e.

$$
\{f, g\}_{R}=\left.\{F, G\}_{D}\right|_{\mathcal{S}}
$$

where $F, G: \mathcal{M} \rightarrow \mathbb{R}$ are two functions such that $f=\left.F\right|_{\mathcal{S}}$ and $g=\left.G\right|_{\mathcal{S}}$. Of course, an identical construction can be induced on any leaf $\mathcal{F}_{s}$ from the foliation $\mathcal{F}$. There arises, of course, a question how 'robust' this construction is, i.e. to what extent the obtained deformation $\pi_{D}$ and the reduction $\pi_{R}$ are 
independent of the choice of particular functions $\varphi_{i}$ that define our submanifold $S$. This issue will be partially addressed in next sections.

The results of this section as well as the results presented in Sections 4 and 5 suggest that the concept of the classification of constraints as being either of "first-class" or of "second-class", proposed by Dirac, should be reexamined when one looks on the problem from the point of view of Poisson geometry. First of all it is clear that the procedure called Dirac reduction has two different levels. The first level we call Dirac deformation as we deform a Poisson bivector $\pi$ from manifold $\mathcal{M}$ to another Poisson bivector $\pi_{D}$ on the same manifold $\mathcal{M}$. A sufficient condition for the existence of $\pi_{D}$ for a given set of constraints $\varphi_{i}$, $i=1, \ldots, m$ is a nondegeneracy of the Gram matrix $\varphi$. From the construction all constraints $\varphi_{i}$ are Casimirs of $\pi_{D}$. The second level of the construction we call Dirac restriction as we restrict a Poisson bivector $\pi_{D}$ to its symplectic leaf $\mathcal{F}_{s}$. In the original Dirac construction it was a particular one, namely $\mathcal{F}_{0}=\mathcal{S}$. A Poisson bivector on $\mathcal{F}_{s}$ is denoted by $\pi_{R}$. For the existence of the second step additional restrictions on $\varphi_{i}$ have to be imposed. Actually, $\pi_{R}$ has to be nonsingular. In a standard classification it means that we have to exclude constraints of first-class. Let us remind that a constraint $\varphi_{k}$ is of first class if its Poisson bracket with all the remaining constants $\varphi_{i}$ vanishes on $\mathcal{S}$, that is if

$$
\left.\left\{\varphi_{k}, \varphi_{i}\right\}_{\pi}\right|_{\mathcal{S}}=0, \quad i=1, \ldots, m .
$$

Otherwise $\varphi_{k}$ is of second-class. In general, first-class constraints make $\pi_{R}$ singular so that the Dirac reduction procedure can not be performed. Nevertheless, the condition (10) seems to be too strong. In sections 4 and 5 we demonstrate situations where constraints are of first-class but the singularity is 'removable' and so the Poisson bivector $\pi_{R}$ is well defined. It suggests that the classification of constraints given by Dirac should be reformulated in the context of Poisson pencils and Poisson geometry.

\section{Separable quasi-bi-Hamiltonian chains of Be- nenti type}

In the following section we briefly remind basic facts about separable Hamiltonian systems on Riemannian manifolds, which form a special class of quasibi-Hamiltonian chains [3], 11, known also as the so called Benenti systems 21], 22]. Let $(Q, g)$ be a Riemannian manifold with covariant metric tensor $g=\left(g_{i j}\right)$ and with the inverse (contravariant metric tensor) $g^{-1}=G=\left(G^{i j}\right)$. Let $\left(q^{1}, \ldots, q^{n}\right)$ be some coordinate system on $Q$ and let $\left(q^{1}, \ldots, q^{n}, p_{1}, \ldots, p_{n}\right)$ be the corresponding canonical coordinates on the phase space $\mathcal{N}=T^{*} Q$ with the associated Poisson tensor

$$
\theta_{0}=\left(\begin{array}{cc}
0_{n} & I_{n} \\
-I_{n} & 0_{n}
\end{array}\right)
$$

where $I_{n}$ is $n \times n$ unit matrix and $0_{n}$ is the $n \times n$ matrix with all entries equal to zero. Let us consider the Hamiltonian $E: \mathcal{N} \rightarrow \mathbb{R}$ for the geodesic flow on 
$Q:$

$$
E=E(q, p)=\sum_{i, j=1}^{n} G^{i j}(q) p_{i} p_{j}
$$

As it is known, a $(1,1)$-type tensor $B=\left(B_{j}^{i}\right)$ (or a $(2,0)$-type tensor $B=$ $\left.\left(B^{i j}\right)\right)$ is called a Killing tensor with respect to $g$ if $\left\{\sum(B G)^{i j} p_{i} p_{j}, E\right\}_{\theta_{0}}=0$ (or $\left\{\sum(B)^{i j} p_{i} p_{j}, E\right\}_{\theta_{0}}=0$ ). An important generalization of this notion is formulated in the following definition.

Definition 4 Let $L=\left(L_{j}^{i}\right)$ be a second order mixed type (i.e. $(1,1)$-) tensor on $Q$ and let $\bar{L}: \mathcal{N} \rightarrow \mathbb{R}$ be a function on $\mathcal{N}$ defined as $\bar{L}=\frac{1}{2} \sum_{i, j=1}^{n}(L G)^{i j} p_{i} p_{j}$, where $L G$ is a $(1,1)$ tensor with components $(L G)^{i j}=\sum_{i, j=1}^{n} L_{k}^{i} g^{k j}$. If

$$
\{\bar{L}, E\}_{\theta_{0}}=\alpha E, \quad \text { where } \quad \alpha=\sum_{i, j=1}^{n} G^{i j} \frac{\partial f}{\partial q^{i}} p_{j}, \quad f=\operatorname{Tr}(L),
$$

then $L$ is called a special conformal Killing tensor with the associated potential $f=\operatorname{Tr}(L)[11]$.

The importance of this notion lies in the fact that on manifolds with tensor $L$ the geodesic flows are separable. There exist, in this case, $n$ constants of motion, quadratic in momenta, of the form

$$
\begin{gathered}
E_{r}=\sum_{i, j=1}^{n} A_{r}^{i j} p_{i} p_{j}=\sum_{i, j=1}^{n}\left(K_{r} G\right)^{i j} p_{i} p_{j}, \quad r=1, \ldots, n, \\
\left(K_{r} G\right)^{i j}=\sum_{k=1}^{n}\left(K_{r}\right)_{k}^{i} G^{k j}
\end{gathered}
$$

where $A_{r}$ and $K_{r}$ are Killing tensors of type $(2,0)$ and $(1,1)$, respectively. Moreover, all the Killing tensors $K_{r}$ are given by the following 'cofactor' formula

$$
\operatorname{cof}(\xi I-L)=\sum_{i=1}^{n-1} K_{n-i} \xi^{i},
$$

where $\operatorname{cof}(A)$ stands for the matrix of cofactors, so that $\operatorname{cof}(A) A=(\operatorname{det} A) I$. Notice that $K_{1}=I$, hence $A_{1}=G$ and $E_{1} \equiv E$. Since the tensors $K_{r}$ are Killing, with a common set of eigenfunctions, the functions $E_{r}$ satisfy $\left\{E_{s}, E_{r}\right\}_{\theta_{0}}=0$ and thus they constitute a system of $n$ constants of motion in involution with respect to the Poisson structure $\theta_{0}$. So, for a given metric tensor $g$, the existence of a special conformal Killing tensor $L$ is a sufficient condition for the geodesic flow on $\mathcal{N}$ to be a Liouville integrable Hamiltonian system. 
The special conformal Killing tensor $L$ can be lifted from $Q$ to a $(1,1)$-type tensor on $\mathcal{N}=T^{*} Q$ where it takes the form

$$
N=\left(\begin{array}{cc}
L & 0_{n} \\
F & L^{T}
\end{array}\right), \quad F_{j}^{i}=\frac{\partial}{\partial q^{i}}(L p)_{j}-\frac{\partial}{\partial q^{j}}\left(p^{T} L\right)_{i} .
$$

The lifted $(1,1)$ tensor $N$ is called a recursion operator. An important property of $N$ is that when it acts on the canonical Poisson tensor $\theta_{0}$ it produces another Poisson tensor

$$
\theta_{1}=N \theta_{0}=\left(\begin{array}{cc}
0 & L \\
-L^{T} & F
\end{array}\right)
$$

compatible with the canonical one (actually $\theta_{0}$ is compatible with $N^{k} \theta_{0}$ for any integer $k$ ).

It is now possible to show that the geodesic Hamiltonians $E_{r}$ satisfy on $\mathcal{N}=T^{*} Q$ the set of relations

$$
\theta_{0} d E_{r+1}=\theta_{1} d E_{r}+\rho_{r} \theta_{0} d E_{1}, \quad E_{n+1}=0, \quad r=1, \ldots, n,
$$

where the functions $\rho_{r}(q)$ are coefficients of the characteristic polynomial of $L$ (i.e. minimal polynomial of $N$ ), which is a special case of the quasi-biHamiltonian chain (3) [1].

It turns out that with the tensor $L$ we can (generically) associate a coordinate system on $\mathcal{N}$ in which the flows associated with all the functions $E_{r}$ separate. Namely, let $\left(\lambda^{1}(q), \ldots, \lambda^{n}(q)\right)$ be $n$ distinct, functionally independent eigenvalues of $L$, i.e. solutions of the characteristic equation $\operatorname{det}(\xi I-L)=0$. Solving these relations with respect to $q$ we get the transformation $\lambda \rightarrow q$

$$
q^{i}=\alpha_{i}(\lambda), \quad i=1, \ldots, n .
$$

The remaining part of the transformation to the separation coordinates can be obtained as a canonical transformation reconstructed from the generating function $W(p, \lambda)=\sum_{i} p_{i} \alpha_{i}(\lambda)$ in the standard way by solving the implicit relations $\mu_{i}=\frac{\partial W(p, \lambda)}{\partial \lambda^{2}}$ with respect to $p_{i}$ obtaining $p_{i}=\beta_{i}(\lambda, \mu)$. In the $(\lambda, \mu)$ coordinates, known as the Darboux-Nijenhuis coordinates (DN), the tensor $L$ is diagonal $L=\operatorname{diag}\left(\lambda^{1}, \ldots, \lambda^{n}\right) \equiv \Lambda_{n}$, while the Hamiltonians (13) of our quasibi-Hamiltonian chain attain the form 3

$$
E_{r}(\lambda, \mu)=-\sum_{i=1}^{n} \frac{\partial \rho_{r}}{\partial \lambda^{i}} \frac{f_{i}\left(\lambda^{i}\right) \mu_{i}^{2}}{\Delta_{i}} \quad r=1, \ldots, n,
$$

where

$$
\Delta_{i}=\prod_{k=1, \ldots n, k \neq i}\left(\lambda^{i}-\lambda^{k}\right),
$$

$\rho_{r}(\lambda)$ are symmetric polynomials (Viéte polynomials) defined by the relation

$$
\operatorname{det}(\xi I-\Lambda)=\left(\xi-\lambda^{1}\right)\left(\xi-\lambda^{2}\right) \ldots\left(\xi-\lambda^{n}\right)=\sum_{r=0}^{n} \rho_{r} \xi^{r},
$$


and where $f_{i}$ are arbitrary smooth functions of one real argument.

It turns out that there exists a sequence of generic separable potentials $V_{r}^{(k)}$, $k \in \mathbf{Z}$, which can be added to geodesic Hamiltonians $E_{r}$ such that the new Hamiltonians

$$
H_{r}(q, p)=E_{r}(q, p)+V_{r}^{(k)}(q), \quad r=1, \ldots, n,
$$

are still separable in the same coordinates $(\lambda, \mu)$. These generic potentials are given by some recursion relations [4, [10]. The Hamiltonians $H_{r}: \mathcal{N} \rightarrow \mathbb{R}$ in (19) satisfy the following quasi-bi-Hamiltonian chain

$$
\theta_{0} d H_{r+1}=\theta_{1} d H_{r}+\rho_{r} \theta_{0} d H_{1}, \quad H_{n+1}=0, \quad r=1, \ldots, n .
$$

In the DN coordinates the Hamiltonians $H_{r}$ attain the form 3 ]

$$
H_{r}(\lambda, \mu)=-\sum_{i=1}^{n} \frac{\partial \rho_{r}}{\partial \lambda^{i}} \frac{f_{i}\left(\lambda^{i}\right) \mu_{i}^{2}+\gamma_{i}\left(\lambda^{i}\right)}{\Delta_{i}}, \quad r=1, \ldots, n,
$$

where potentials $V_{r}^{(k)}$ enter $H_{r}$ as $\gamma_{i}\left(\lambda^{i}\right)=\left(\lambda^{i}\right)^{n+k-1}$. From (21) it immediately follows that in $(\lambda, \mu)$ variables the contravariant metric tensor $G$ and all the Killing tensors $K_{r}$ are diagonal

$$
G^{i j}=\frac{f_{i}\left(\lambda^{i}\right)}{\Delta_{i}} \delta^{i j}, \quad\left(K_{r}\right)_{j}^{i}=-\frac{\partial \rho_{r}}{\partial \lambda^{i}} \delta_{j}^{i} .
$$

Moreover, in the $(\lambda, \mu)$ coordinates the recursion operator and the tensor $\theta_{1}$ attain the form

$$
N=\left(\begin{array}{cc}
\Lambda_{n} & 0_{n} \\
0_{n} & \Lambda_{n}
\end{array}\right), \quad \theta_{1}=\left(\begin{array}{cc}
0_{n} & \Lambda_{n} \\
-\Lambda_{n} & 0_{n}
\end{array}\right)
$$

while $\theta_{0}$ remains in the form (11) since the transformation $(q, p) \rightarrow(\lambda, \mu)$ is canonical.

The quasi-bi-Hamiltonian chain (20) on $\mathcal{N}$ can easily be lifted to a biHamitonian chain (2) on the extended manifold $\mathcal{M}=T^{*} Q \times \mathbf{R}=\mathcal{N} \times \mathbf{R}$ 11.

Having such a complete picture of separable quasi-bi-Hamiltonian chains on Riemannian manifolds, one can ask a question: what kind of holonomic constraints can be imposed on considered systems so that this quasi-bi-Hamiltonian separability schema is preserved? The simplest admissible case of such constraints will be considered in the next sections.

\section{Reduction $\lambda^{n}=0$}

Let us consider a particle moving in our Riemannian manifold $Q$ equipped with the coordinates $\left(q^{1}, \ldots, q^{n}\right)$. Suppose that this particle is subordinated to some holonomic constraints on $Q$ defined by the set of relations

$$
\varphi_{k}(q)=0, \quad k=1, \ldots, r
$$


that define some submanifold of $Q$. The velocity $v=\sum_{i} v^{i} \frac{\partial}{\partial q^{2}}$ of this particle must then remain tangent to this submanifold so that

$$
0=\left\langle d \varphi_{k}, v\right\rangle=\sum_{i=1}^{n} \frac{\partial \varphi_{k}}{\partial q^{i}} v^{i} .
$$

and thus in our coordinates $v^{i}=\sum_{j} G^{i j} p_{j}$ the motion of the particle in the phase space $\mathcal{N}=T^{*} Q$ is constrained not only by the $r$ relations (22) but also by the $r$ relations

$$
\varphi_{r+k}(q, p) \equiv \sum_{i, j=1}^{n} G^{i j}(q) \frac{\partial \varphi_{k}(q)}{\partial q^{i}} p_{j}=0, \quad k=1, \ldots, r .
$$

that are nothing else than the lift of (22) to $\mathcal{N}$. We will call the constraints (23) a $g$-consequence of the constraints (22), as they are natural differential consequences of (22) at given metric tensor $g$. The constraints (22)-(23) define a submanifold $\mathcal{S}$ of $\mathcal{N}$ of dimension $n-2 r$.

Let us now consider our quasi-bi-Hamiltonian chain (20) in $\mathcal{N}$. We would like to know what types of holonomic constraints (22) on $Q$ do not destroy the separability of the constrained chain? This is a complicated question and due to its nature it is most convenient (for a moment) to consider it directly in our separation coordinates $(\lambda, \mu)$. Thus, in this section we will analyze only a very special choice of the functions $\varphi_{k}$ in (22). Namely, we put $r=1$ (so that $n-2 r=n-2$ which corresponds to $m=2$ in Section 2) and define the corresponding function $\varphi_{1}^{\prime}$ in $(\lambda, \mu)$ variables as

$$
\varphi_{1}^{\prime}(\lambda)=\lambda^{n}
$$

Since the metric tensor $G=\left(G^{i j}\right)$ has in $(\lambda, \mu)$ coordinates the diagonal form $G=\operatorname{diag}\left(f_{i}\left(\lambda^{i}\right) / \Delta_{i}\right), i=1, \ldots, n$ and since the equation (23) has an invariant form (i.e. in $(\lambda, \mu)$ coordinates it has the same form with $q$ and $p$ replaced by $\lambda$ and $\mu$ respectively) the $g$-consequence of (24) reads as

$$
\varphi_{2}^{\prime}(\lambda, \mu)=\frac{f_{n}\left(\lambda^{n}\right)}{\Delta_{n}} \mu_{n}
$$

(we use' here since soon we will modify the constraints (24)-(25) to a simpler form). These two constraints define a subset $\mathcal{S}^{\prime}=\left\{\varphi_{1}^{\prime}=0, \varphi_{2}^{\prime}=0\right\}$ of $\mathcal{N}$. We will however restrict us to a submanifold $\mathcal{S}=\left\{\lambda^{n}=0, \mu_{n}=0\right\}$ neglecting the term $f_{n}\left(\lambda^{n}\right) / \Delta_{n}$ in $\varphi_{2}^{\prime}$ (for $\Delta_{n} \neq 0 \mathcal{S} \subset \mathcal{S}^{\prime}$ and in case of the systems when $f_{n}$ never vanishes $\mathcal{S}$ and $\mathcal{S}^{\prime}$ coincide). The reason for this is that various calculations with the help of these new constraints $\lambda^{n}=0, \mu_{n}=0$ are simpler. Let us now perform the Dirac reduction of our Poisson operators $\theta_{0}$ and $\theta_{1}$

$$
\theta_{0}=\left(\begin{array}{cc}
0_{n} & I_{n} \\
-I_{n} & 0_{n}
\end{array}\right) \quad, \quad \theta_{1}=\left(\begin{array}{cc}
0_{n} & \Lambda_{n} \\
-\Lambda_{n} & 0_{n}
\end{array}\right)
$$


and of the corresponding quasi-bi-Hamiltonian chain (16) (geodesic case) or (20) (potential case) on the submanifold $\mathcal{S}$. We can do it either by using the constraints (24)- 25 or by the constraints that directly describe $\mathcal{S}$ :

$$
\varphi_{1}(\lambda) \equiv \lambda^{n}=0 \quad, \varphi_{2}(\lambda, \mu) \equiv \mu_{n}=0
$$

(of course $\varphi_{1} \equiv \varphi_{1}^{\prime}$ ). It turns out that the corresponding Dirac deformations (given by (8) or equivalently by (9) ) $\theta_{0, D}$ and $\theta_{0, D}^{\prime}$ of $\theta_{0}$ are not equal. Similarly, $\theta_{1, D} \neq \theta_{1, D}^{\prime}$. However, after reducing $\theta_{0, D}$ and $\theta_{0, D}^{\prime}$ on $\mathcal{S}$ we obtain the same Poisson operator (and similarly with $\theta_{1, D}$ and $\theta_{1, D}^{\prime}$ ).

Theorem 5 With the notation as above, the reduced operators $\theta_{i, R}$ and $\theta_{i, R}^{\prime}$ on $\mathcal{S}$ satisfy $\theta_{0, R}^{\prime}=\theta_{0, R}$ and $\theta_{1, R}^{\prime}=\theta_{1, R}$. If we parametrize $\mathcal{S}$ by the variables $\left(\lambda^{1}, \ldots, \lambda^{n-1}, \mu_{1}, \ldots, \mu_{n-1}\right)$ then

$$
\theta_{0, R}=\left(\begin{array}{cc}
0_{n-1} & I_{n-1} \\
-I_{n-1} & 0_{n-1}
\end{array}\right) \quad, \theta_{1, R}=\left[\begin{array}{cc}
0_{n-1} & \Lambda_{n-1} \\
-\Lambda_{n-1} & 0_{n-1}
\end{array}\right]
$$

with $\Lambda_{n-1}=\operatorname{diag}\left(\lambda^{1}, \ldots, \lambda^{n-1}\right)$.

Proof. Let us start by calculating $\theta_{0, D}$. In this case the $2 \times 2$ Gram matrix $\varphi=\left(\left\{\varphi_{i}, \varphi_{j}\right\}_{\theta_{0}}\right)$ is of the form

$$
\varphi=\left[\begin{array}{cc}
0 & 1 \\
-1 & 0
\end{array}\right]
$$

and thus according to (7) we have $Z_{1}=X_{2}$ and $Z_{2}=-X_{1}$. The formula (8) yields

$$
\theta_{0, D}=\theta_{0}-X_{1} \wedge X_{2}=\theta_{0}-\frac{\partial}{\partial \lambda^{n}} \wedge \frac{\partial}{\partial \mu_{n}}=\sum_{i=1}^{n-1} \frac{\partial}{\partial \lambda^{i}} \wedge \frac{\partial}{\partial \mu_{i}}
$$

since $X_{1}=\theta_{0} d \varphi_{1}=-\partial / \partial \mu_{n}$ and $X_{2}=\theta_{0} d \varphi_{2}=\partial / \partial \lambda^{n}$. So, in $(\lambda, \mu)$ coordinates we have

$$
\theta_{0, D}=\left[\begin{array}{ccc}
\multicolumn{2}{c|}{0_{n-1}} & 0_{(n-1) \times 1} \\
\hline \multicolumn{2}{c|}{0_{1 \times(n-1)}} & 0 \\
\hline I_{n-1} & 0_{(n-1) \times 1} & \multicolumn{2}{|c}{0_{n}} \\
0_{1 \times(n-1)} & 0 & \multicolumn{2}{|c}{}
\end{array}\right]
$$

The operator (29) has thus the $n$-th and the last column and the $n$-th and the last row filled with zeros. These two rows and two columns of zeros correspond to the fact (see Theorem (1) that the constraints $\varphi_{1}=\lambda^{n}$ and $\varphi_{2}=\mu_{n}$ are now Casimir functions for $\theta_{0, D}$. We can thus directly project $\theta_{0, D}$ onto the symplectic leaf $\mathcal{S}=\left\{\lambda^{n}=\mu_{n}=0\right\}$ of $\theta_{0, D}$ by simply removing the two zero columns and zero rows from (29), which yield the operator $\theta_{0, R}$ that written in coordinates $\left(\lambda^{1}, \ldots \lambda^{n-1}, \mu_{1}, \ldots \mu_{n-1}\right)$ on $\mathcal{S}$ attains the form as in (28). Similar computations show that in the case of $\theta_{1, D}$

$$
\varphi=\left[\begin{array}{cc}
0 & \lambda^{n} \\
-\lambda^{n} & 0
\end{array}\right]=\left[\begin{array}{cc}
0 & \varphi_{1} \\
-\varphi_{1} & 0
\end{array}\right]
$$


and thus (see (10) $) \varphi_{1}$ and $\varphi_{2}$ are first-class constraints. Indeed, following (7) we have $Z_{1}=\frac{1}{\lambda^{n}} X_{2}$ and $Z_{2}=-\frac{1}{\lambda^{n}} X_{1}$ and the formula (8) yields

$$
\theta_{1, D}=\theta_{1}-\frac{1}{\lambda^{n}} X_{1} \wedge X_{2}
$$

which seems to be singular on $\mathcal{S}$. Nevertheless this singularity is 'removable' since $X_{1}=\theta_{1} d \varphi_{1}=-\lambda^{n} \partial / \partial \mu_{n}, X_{2}=\theta_{1} d \varphi_{2}=\lambda^{n} \partial / \partial \lambda^{n}$ and hence

$$
\theta_{1, D}=\theta_{1}-\lambda^{n} \frac{\partial}{\partial \lambda^{n}} \wedge \frac{\partial}{\partial \mu_{n}}=\sum_{i=1}^{n-1} \lambda^{i} \frac{\partial}{\partial \lambda^{i}} \wedge \frac{\partial}{\partial \mu_{i}}
$$

So, the matrix form of $\theta_{1, D}$ in $(\lambda, \mu)$ coordinates becomes

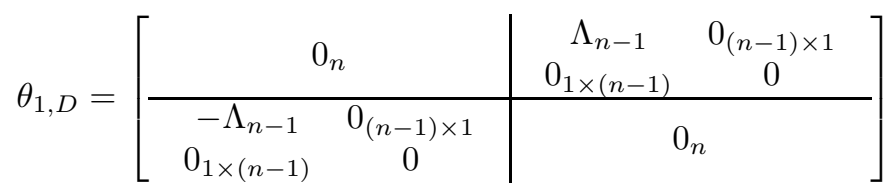

and the projection of this operator onto the symplectic leaf $\mathcal{S}=\left\{\lambda^{n}=\mu_{n}=0\right\}$ of $\theta_{1, D}$ yields exactly $\theta_{1, R}$ as in (28).

Passing to $\varphi_{1}^{\prime}, \varphi_{2}^{\prime}$ notice that

$$
\varphi_{12}^{\prime}=\left\{\varphi_{1}^{\prime}, \varphi_{2}^{\prime}\right\}_{\theta_{0}}=\frac{f_{n}\left(\lambda^{n}\right)}{\Delta_{n}}
$$

so one can have a situation when $\left.\varphi_{12}^{\prime}\right|_{\mathcal{S}}=0$ which again leads to first-class constraints according to Dirac classification. Nevertheless, calculations similar to these for $\varphi_{1}, \varphi_{2}$ show that singularities are again 'removable' and we end up with the same form of $\theta_{0, D}$ and $\theta_{1, D}$.

Of course, the reduced operators $\theta_{0, R}$ and $\theta_{1, R}$ are compatible. Moreover, it is easy to see that the Hamiltonians (21) restricted to $\mathcal{S}$ become

$$
\begin{gathered}
H_{r, R}\left(\lambda^{1}, \ldots \lambda^{n-1}, \mu_{1}, \ldots \mu_{n-1}\right) \\
=\left.H_{r}\right|_{\mathcal{S}}=-\sum_{i=1}^{n-1} \frac{\partial \rho_{r, R}}{\partial \lambda^{i}} \frac{f_{i, R}\left(\lambda^{i}\right) \mu_{i}^{2}+\gamma_{i, R}\left(\lambda^{i}\right)}{\Delta_{i, R}}, \quad r=1, \ldots, n-1, \\
H_{n, R}=\left.H_{n}\right|_{\mathcal{S}}=0
\end{gathered}
$$

where $f_{i, R}\left(\lambda^{i}\right)=f_{i}\left(\lambda^{i}\right) / \lambda^{i}, \gamma_{i, R}\left(\lambda^{i}\right)=\gamma_{i}\left(\lambda^{i}\right) / \lambda^{i}, i=1, \ldots, n-1$ and $\rho_{r, R}(\lambda)$ are Viéte polynomials of dimension $n-1$. Having all this is mind, we can formulate the following corollary.

Corollary 6 Both Dirac reductions (24)-(25) and (27) of the quasi-bi-Hamiltonian system (20) onto $\mathcal{S}$ lead to the same quasi-bi-Hamiltonian system of the form

$$
\theta_{0, R} d H_{r+1, R}=\theta_{1, R} d H_{r, R}+\rho_{r, R} \theta_{0, R} d H_{1, R}, \quad H_{n, R}=0, \quad r=1, \ldots, n-1 .
$$

which is thus separable in the variables $\left(\lambda^{1}, \ldots \lambda^{n-1}, \mu_{1}, \ldots \mu_{n-1}\right)$ that coincide on $\mathcal{S}$ with 'first' $n-2$ separation coordinates from our coordinate system $(\lambda, \mu)$ that separates (20). 
Because the reduced quasi-bi-Hamiltonian chain has exactly the form (20), it can be put into a bi-Hamiltonian chain on $\mathcal{S} \times \mathbb{R}$.

Of course this procedure of Dirac reduction, in principle could be performed directly in $(q, p)$ coordinates, since the formula (8) has a tensor character and thus yields the same result no matter what coordinate system we choose for actual calculating of deformations $\theta_{0, D}$ and $\theta_{1, D}$. There is however a major obstacle here: we are usually not able to express the constraint $\lambda^{n}=0$ in 'physical' coordinates $(q, p)$, as equations (17) are noninvertible in general, i.e. there is no algebraic way of solving them with respect to $\lambda$ (if we could, then the second constraint (25) could be computed as the $g$-consequence of the first one calculated directly in $(q, p)$ coordinates, with the help of (23)). Thus, although the picture presented in this section is clear, nevertheless it is somehow useless once we are given the quasi-bi-Hamiltonian chain in natural $(q, p)$ coordinates. In the next section we will demonstrate how this problem can be defused by the use of the so called Hankel-Frobenius coordinates.

\section{Reduction in Hankel-Frobenius coordinates}

As it was demonstrated in the previous section, the constraints $\varphi_{1}=\lambda^{n}, \varphi_{2}=$ $\mu_{n}$ preserve the separability on the constrained submanifold $\mathcal{S}$ but are very inconvenient to handle with, as in general we do not know how to express them in the original coordinates $(q, p)$. More convenient for this purpose is the set of so called Hankel-Frobenius coordinates $\left(\rho_{i}, v_{i}\right)_{i=1}^{n}$ 24, 25]. They are noncanonical coordinates, related to the separated coordinates in the following way

$$
\begin{aligned}
& \rho_{i}=\rho_{i}(\lambda), \quad i=1, \ldots, n, \\
& v_{i}=\sum_{j}\left(V_{n}^{-1}\right)_{i j} \mu_{j}=\sum_{k} \frac{\partial \rho_{i}}{\partial \lambda^{k}} \frac{1}{\Delta_{k}} \mu_{k}, \quad i=1, \ldots, n,
\end{aligned}
$$

where

$$
V_{n}=\left(\begin{array}{cccc}
\left(\lambda^{1}\right)^{n-1} & \cdots & \lambda^{1} & 1 \\
\vdots & \vdots & \vdots & \vdots \\
\left(\lambda^{n}\right)^{n-1} & \cdots & \lambda^{n} & 1
\end{array}\right)
$$

is a Vandermonde matrix and $\rho_{i}$ are Viéte polynomials (18). The mapping (30) is not a point transformation on $Q$ and therefore it makes no sense to distinguish covariant and contravariant indices now. Applying the map (30) we obtain the form of operators $\theta_{0}$ and $\theta_{1}$ and the $2 n$-dimensional recursion operator $N_{n}$ (15) in $(\rho, v)$ coordinates:

$$
\begin{gathered}
\theta_{0}=\left(\begin{array}{cc}
0 & U_{n} \\
-U_{n} & 0
\end{array}\right), \quad U_{n}=\left(\begin{array}{cccc}
0 & 0 & \cdots & 1 \\
0 & \cdots & 1 & \rho_{1} \\
\vdots & \cdots & \cdots & \vdots \\
1 & \rho_{1} & \cdots & \rho_{n-1}
\end{array}\right), \\
\theta_{1}=N_{n} \theta_{0}=\left(\begin{array}{cc}
0 & F_{n} U_{n} \\
-F_{n} U_{n} & 0
\end{array}\right), \quad F_{n} U_{n}=U_{n} F_{n}^{T} .
\end{gathered}
$$




$$
N_{n}=\left(\begin{array}{cc}
F_{n} & 0 \\
0 & F_{n}
\end{array}\right), \quad F_{n}=\left(\begin{array}{ccccc}
-\rho_{1} & 1 & \cdots & \cdots & 0 \\
-\rho_{2} & 0 & 1 & \cdots & 0 \\
\vdots & \vdots & & & \vdots \\
-\rho_{n-1} & 0 & \cdots & \cdots & 1 \\
-\rho_{n} & 0 & \cdots & \cdots & 0
\end{array}\right) .
$$

We will now consider yet another deformation (8) of $\theta_{0}$ and $\theta_{1}$, given in $(\lambda, \mu)$ variables by the constraints

$$
\begin{aligned}
\varphi_{1}^{\prime \prime} & =\rho_{n}(\lambda)=(-1)^{n} \lambda^{1} \lambda^{2} \cdots \lambda^{n} \\
\varphi_{2}^{\prime \prime} & =v_{n}(\lambda)=(-1)^{n} \sum_{j=1}^{n} \frac{\mu_{j}}{\Delta_{j}} \lambda^{1} \lambda^{2} \cdots \lambda^{j-1} \lambda^{j+1} \cdots \lambda^{n}
\end{aligned}
$$

equal to last pair of Hankel-Frobenius coordinates. The constraints (33) are related with the constraints (27) as

$$
\begin{aligned}
& \varphi_{1}^{\prime \prime}=\psi_{1} \varphi_{1} \\
& \varphi_{2}^{\prime \prime}=\psi_{2} \varphi_{1}+\psi_{3} \varphi_{2}
\end{aligned}
$$

where the functions

$$
\begin{aligned}
& \psi_{1}=(-1)^{n} \lambda^{1} \lambda^{2} \cdots \lambda^{n-1} \\
& \psi_{2}=(-1)^{n} \sum_{j=1}^{n-1} \frac{\mu_{j}}{\Delta_{j}} \lambda^{1} \lambda^{2} \cdots \lambda^{j-1} \lambda^{j+1} \cdots \lambda^{n-1}
\end{aligned}
$$

and $\psi_{3}=(-1)^{n} \lambda^{1} \lambda^{2} \cdots \lambda^{n-1} / \Delta_{n}$ never vanish at $\mathcal{S}=\left\{\varphi_{1}=\varphi_{2}=0\right\} \quad$ (in fact, $\left.\psi_{2}\right|_{\mathcal{S}}=-1$ and $\left.\left.\psi_{3}\right|_{\mathcal{S}}=-\left(\mu_{1}+\ldots+\mu_{n-1}\right)\right)$ and thus the constraints (33) define (locally) the same submanifold $\mathcal{S}$ as the constraints (27). The corresponding deformations $\theta_{0, D}^{\prime \prime}$ and $\theta_{1, D}^{\prime \prime}$ of $\theta_{0}$ and $\theta_{1}$ will of course not be equal to $\theta_{0, D}$ and $\theta_{1, D}$, but again it turns out that their reductions on $S$ will coincide with the corresponding reductions of $\theta_{0, D}$ and $\theta_{1, D}$ on $\mathcal{S}$.

Theorem 7 In the notation as above, $\theta_{0, R}^{\prime \prime}=\theta_{0, R}$ and $\theta_{0, R}^{\prime \prime}=\theta_{0, R}$

Proof. We will use (9) rather than (8) since it turns out that the calculations are in this case simpler when one uses bracket definition of Dirac deformation than bivector definition. Applying (9), we easily get that for any two functions $A, B: \mathcal{N} \rightarrow R$

$$
\{A, B\}_{\theta_{0, D}}=\{A, B\}_{\theta_{0}}+\frac{\left\{A, \varphi_{2}\right\}_{\theta_{0}}\left\{B, \varphi_{1}\right\}_{\theta_{0}}-\left\{A, \varphi_{1}\right\}_{\theta_{0}}\left\{B, \varphi_{2}\right\}_{\theta_{0}}}{\left\{\varphi_{1}, \varphi_{2}\right\}_{\theta_{0}}}
$$

where of course $\left\{\varphi_{1}, \varphi_{2}\right\}_{\theta_{0}}=1$ and so it does not vanish on $\mathcal{S}$. Similarly

$$
\{A, B\}_{\theta_{0, D}^{\prime \prime}}=\{A, B\}_{\theta_{0}}+\frac{\left\{A, \varphi_{2}^{\prime \prime}\right\}_{\theta_{0}}\left\{B, \varphi_{1}^{\prime \prime}\right\}_{\theta_{0}}-\left\{A, \varphi_{1}^{\prime \prime}\right\}_{\theta_{0}}\left\{B, \varphi_{2}^{\prime \prime}\right\}_{\theta_{0}}}{\left\{\varphi_{1}^{\prime \prime}, \varphi_{2}^{\prime \prime}\right\}_{\theta_{0}}}
$$


Using the relations (34) between the deformed constraints $\varphi_{i}^{\prime \prime}$ and the original constraints $\varphi_{i}$, the Leibniz property of Poisson brackets and the fact that $\psi_{1}$ and $\psi_{3}$ depend only on $\lambda$, we obtain

$$
\begin{aligned}
\left\{\varphi_{1}^{\prime \prime}, \varphi_{2}^{\prime \prime}\right\}_{\theta_{0}} & =\psi_{1} \varphi_{1}\left\{\varphi_{1}, \psi_{2}\right\}_{\theta_{0}}+\varphi_{1}^{2}\left\{\psi_{1}, \psi_{2}\right\}_{\theta_{0}}+ \\
& +\psi_{1} \psi_{3}\left\{\varphi_{1}, \varphi_{2}\right\}_{\theta_{0}}+\varphi_{1} \varphi_{2}\left\{\psi_{1}, \psi_{3}\right\}_{\theta_{0}}
\end{aligned}
$$

so that $\left.\left\{\varphi_{1}^{\prime \prime}, \varphi_{2}^{\prime \prime}\right\}_{\theta_{0}}\right|_{\mathcal{S}}=\left.\psi_{1} \psi_{3}\left\{\varphi_{1}, \varphi_{2}\right\}_{\theta_{0}}\right|_{\mathcal{S}}=\left.\psi_{1} \psi_{3}\right|_{\mathcal{S}}$. Similar calculations show that

$$
\begin{aligned}
& \left.\left(\left\{A, \varphi_{2}^{\prime \prime}\right\}_{\theta_{0}}\left\{B, \varphi_{1}^{\prime \prime}\right\}_{\theta_{0}}-\left\{A, \varphi_{1}^{\prime \prime}\right\}_{\theta_{0}}\left\{B, \varphi_{2}^{\prime \prime}\right\}_{\theta_{0}}\right)\right|_{\mathcal{S}} \\
& =\left.\psi_{1} \psi_{3}\left(\left\{A, \varphi_{2}\right\}_{\theta_{0}}\left\{B, \varphi_{1}\right\}_{\theta_{0}}-\left\{A, \varphi_{1}\right\}_{\theta_{0}}\left\{B, \varphi_{2}\right\}_{\theta_{0}}\right)\right|_{\mathcal{S}}
\end{aligned}
$$

and thus the factors $\psi_{1} \psi_{3}$ in the numerator and in the denominator of (35) cancel and we conclude that

$$
\left.\{A, B\}_{\theta_{0, D}^{\prime \prime}}\right|_{\mathcal{S}}=\left.\{A, B\}_{\theta_{0, D}}\right|_{S}
$$

which is the same as to claim that $\theta_{0, R}^{\prime \prime}=\theta_{0, R}$. The proof that $\theta_{1, R}^{\prime \prime}=\theta_{1, R}$ is similar: first one shows that

$$
\left.\left\{\varphi_{1}^{\prime \prime}, \varphi_{2}^{\prime \prime}\right\}_{\theta_{1}}\right|_{\mathcal{S}}=\left.\psi_{1} \psi_{3}\left\{\varphi_{1}, \varphi_{2}\right\}_{\theta_{1}}\right|_{\mathcal{S}}=\left.\psi_{1} \psi_{3}\right|_{\mathcal{S}} \lambda^{n}
$$

which is by the way equal to zero on $\mathcal{S}$. However, we also get

$$
\begin{aligned}
& \left.\left(\left\{A, \varphi_{2}^{\prime \prime}\right\}_{\theta_{1}}\left\{B, \varphi_{1}^{\prime \prime}\right\}_{\theta_{1}}-\left\{A, \varphi_{1}^{\prime \prime}\right\}_{\theta_{1}}\left\{B, \varphi_{2}^{\prime \prime}\right\}_{\theta_{1}}\right)\right|_{\mathcal{S}} \\
& =\left.\psi_{1} \psi_{3}\left(\left\{A, \varphi_{2}\right\}_{\theta_{1}}\left\{B, \varphi_{1}\right\}_{\theta_{1}}-\left\{A, \varphi_{1}\right\}_{\theta_{1}}\left\{B, \varphi_{2}\right\}_{\theta_{1}}\right)\right|_{\mathcal{S}}
\end{aligned}
$$

so that on $\mathcal{S}$

$$
\begin{gathered}
\left.\{A, B\}_{\theta_{1, D}}^{\prime \prime}\right|_{\mathcal{S}}=\left.\{A, B\}_{\theta_{1}}\right|_{\mathcal{S}}+\left.\frac{\psi_{1} \psi_{3}\left(\left\{A, \varphi_{2}\right\}_{\theta_{1}}\left\{B, \varphi_{1}\right\}_{\theta_{1}}-\left\{A, \varphi_{1}\right\}_{\theta_{1}}\left\{B, \varphi_{2}\right\}_{\theta_{1}}\right)}{\psi_{1} \psi_{3}\left\{\varphi_{1}, \varphi_{2}\right\}_{\theta_{1}}}\right|_{\mathcal{S}} \\
=\left.\{A, B\}_{\theta_{1}}\right|_{\mathcal{S}}+\left.\frac{\left\{A, \varphi_{2}\right\}_{\theta_{1}}\left\{B, \varphi_{1}\right\}_{\theta_{1}}-\left\{A, \varphi_{1}\right\}_{\theta_{1}}\left\{B, \varphi_{2}\right\}_{\theta_{1}}}{\lambda^{n}}\right|_{\mathcal{S}}=\left.\{A, B\}_{\theta_{1, D}}\right|_{\mathcal{S}}
\end{gathered}
$$

since the term $\lambda^{n}$ in the last expression does not cause any singularity: for every possible combination $A, B=\lambda^{i}, \mu_{j}$ the numerator in the above expression is either zero or some multiple of $\lambda^{n}$. This proves that $\theta_{1, R}^{\prime \prime}=\theta_{1, R}$.

Before we proceed with the main theme of this article, let us make a digression: we will establish the form of $\theta_{0, R}$ and $\theta_{1, R}$ in $(\rho, v)$-coordinates.

Lemma 8 In $(\rho, v)$-coordinates we have

$$
\begin{aligned}
& \theta_{0, R}=\left(N_{n-1}\right)^{-1}\left(\begin{array}{cc}
0 & U_{n-1} \\
-U_{n-1} & 0
\end{array}\right)=\left(\begin{array}{cc}
0_{n-1} & \left(F_{n-1}\right)^{-1} U_{n-1} \\
-\left(F_{n-1}\right)^{-1} U_{n-1} & 0_{n-1}
\end{array}\right) \\
& \theta_{1, R}=\left(\begin{array}{cc}
0 & U_{n-1} \\
-U_{n-1} & 0
\end{array}\right)
\end{aligned}
$$


This lemma can be proved either by direct calculating of the deformation formula (8) in $(\rho, v)$-coordinates or by transforming both $\theta_{0, R}$ and $\theta_{1, R}$ with the map that is the restriction of the map (30) to $\mathcal{S}$. This restricted map has no longer the form (30) and this is why the operators $\theta_{0, R}$ and $\theta_{1, R}$ does not transform respectively to the operators of the form (31) and (32) with $n$ replaced by $n-1$.

Having established Theorem[7we can now, for a given (quasi)-bi-Hamiltonian system (20), perform the Dirac reduction $\lambda^{n}=\mu_{n}=0$ directly in physical coordinates $(q, p)$ by performing the equivalent reduction $\rho_{n}=v_{n}=0$ provided that we are able to express the constraints (33) directly in physical coordinates. The first of the constraints in (33), i.e. $\rho_{n}=0$ is given directly in $(q, p)$ coordinates once the system (16) has been given (this is the reason for which we consider this constraint and Hankel-Frobenius coordinates). Below we shall show how to express the second constraint $\left(v_{n}=0\right)$ in physical coordinates.

Let us first observe that in case of systems for which $f_{i}\left(\lambda^{i}\right)=$ const (that does not depend on $i$ ) the constraint $v_{n}=0$ calculated in $(\lambda, \mu)$ coordinates is just the $g$-consequence of the first one $\left(\rho_{n}=0\right)$, so that in this particular case we can easily get the function $\phi_{2}^{\prime \prime}(q, p)$ by calculating the expression (23) with an appropriate metric tensor $g$ that can be found in $h_{1}$. When the functions $f_{i}$ are more complicated, we must proceed differently.

Theorem 9 Assume that $f_{i}\left(\lambda^{i}\right)=f\left(\lambda^{i}\right)$, i.e. that the functions $f_{i}$ do not depend on $i$. Let also $F$ be the matrix given by (32). Then the coordinates $v_{i}$ expressed in $(q, p)$ coordinates have the following form

$$
v_{i}(q, p)=\sum_{j=1}^{n}\left(\left(f(F)^{-1}\right)_{i j} \sum_{k, l=1}^{n} G^{k l}(q) \frac{\partial \rho_{j}}{\partial q^{k}} p_{l}\right)
$$

with $F$ being expressed in $q$.

Proof. Let us expand the function $f$ in a formal Laurent series

$$
f(\lambda)=\sum_{m} a_{m} \lambda^{m}, a_{m} \in \mathbb{R}, m \in \mathbf{Z}_{f} \subset \mathbf{Z} .
$$

Let also $v_{k}^{\prime}, k=1, \ldots, n$ be the $g$-consequence of $\rho_{k}$ so that

$$
v_{k}^{\prime}(\lambda, \mu)=\sum_{i=1}^{n} \frac{f_{i}}{\Delta_{i}} \frac{\partial \rho_{k}}{\partial \lambda^{i}} \mu_{i} \text { and } v_{k}^{\prime}(q, p)=\sum_{i, j=1}^{n} G^{i j}(q) \frac{\partial \rho_{k}}{\partial q^{i}} p_{j} .
$$

Setting $\mu=\left(\mu_{1}, \ldots, \mu_{n}\right)^{T}, v=\left(v_{1}, \ldots, v_{n}\right)^{T}, v^{\prime}=\left(v_{1}^{\prime}, \ldots, v_{n}^{\prime}\right)^{T}$ and using the fact (30) that $\mu=V v$ (where $V$ is the Vandermonde matrix) we easily obtain that

$$
v^{\prime}=V^{-1} \operatorname{diag}\left(f\left(\lambda^{1}\right), \ldots, f\left(\lambda^{n}\right)\right) V v
$$

Moreover, from the transformation between $(\lambda, \mu)$ and $(\rho, v)$ coordinates one finds that

$$
V^{-1} \Lambda^{m} V=U\left(F^{T}\right)^{m} U^{-1}=F^{m} .
$$


so that due to (37)

$$
\begin{aligned}
v^{\prime} & =V^{-1} \sum_{m} a_{m} \operatorname{diag}\left(\left(\lambda^{1}\right)^{m}, \ldots,\left(\lambda^{n}\right)^{m}\right) V v=V^{-1}\left(\sum_{m} a_{m} \Lambda^{m}\right) V v \\
& =\left(\sum_{m} a_{m} V^{-1} \Lambda^{m} V\right) v=\left(\sum_{m} a_{m} F^{m}\right) v=f(F) v .
\end{aligned}
$$

This relation is valid in every coordinate system and thus $v(q, p)=f(F(q)) v^{\prime}(q, p)$ which due to (38) yields (36).

In case when the assumption of the theorem is not satisfied, we can not express $v_{n}$ in $(q, p)$ variables using the method presented in the proof. Since in practice this situation is very rare, we choose not to discuss it in this article. Notice that if $f=$ const then $f(F)=$ const $I$ and the formula (36) for $v_{n}$ reduces to the $g$-consequence of $\rho_{n}$, as it should be.

Remark 10 One can ask the question why, in the case of $f \neq$ const, instead of taking $\varphi_{2}^{\prime \prime}=\nu_{n}$ as above, we do not choose $\varphi_{2}^{\prime \prime}$ to be simply the $g$-consequence of $\varphi_{1}^{\prime \prime}=\rho_{n}$, as both pairs of constraints describe the same $\mathcal{S}$. The answer is due to one of the fundamental observations of presented paper, mentioned at the end of section 2. Actually, a constrained submanifold $\mathcal{S}$ can be defined by infinitely many different pairs of constraints, all of them of the first class according to the classical Dirac classification. That is, either the Gram matrix is singular: $\left\{\varphi_{1}^{\prime \prime}, \varphi_{2}^{\prime \prime}\right\}_{\theta}=0$, or $\left.\left\{\varphi_{1}^{\prime \prime}, \varphi_{2}^{\prime \prime}\right\}_{\theta}\right|_{\mathcal{S}}=0$, for one or both $\theta_{i}$, hence, in principle, there is no Dirac deformation $\theta_{i, D}$ or the restriction of $\theta_{i, D}$ on $\mathcal{S}$ is not possible. However, among all these pairs of constraints, there are exceptional pairs, like $\left(\lambda^{n}, \mu_{n}\right)$ or $\left(\rho_{n}, \nu_{n}\right)$, for which singularities are 'removable' and Dirac restricted Poisson pencil is nonsingular. So in fact, these particular constraints have to be considered as second order constraints.

Notice also that in order to determine the constraint $v_{n}$ from (36) we have first to find the function $f$ for example from $E_{1}$, written in $(\lambda, \mu)$ coordinates. So in practice, we cannot avoid the calculation of separation coordinates, and this is the price we have to pay. Nevertheless, in general, it is not difficult to find the transformation (17) and then the geodesic Hamiltonian $E_{1}(\lambda, \mu)$ for the original system. Also, one has to bear in mind that even though the reduction procedure looks trivial in $(\lambda, \mu)$-coordinates, it is usually not possible to express the obtained reduced system back in $(q, p)$-coordinates since our Hamiltonians $E_{r, R}$ have different function $f$ than the Hamiltonians $E_{r}$. That is why we had to use Hankel-Frobenius coordinates.

\section{Examples}

In this chapter we will illustrate the introduced ideas by two examples. In our first example of Dirac reductions of separable systems we will consider the

so called first Newton representation of the seventh-order stationary flow of 
the KdV hierarchy [26, 2]. It is a Lagrangian system of second order Newton equations

$$
\begin{aligned}
& q_{, t t}^{1}=-10\left(q^{1}\right)^{2}+4 q^{2} \\
& q_{, t t}^{2}=-16 q^{1} q^{2}+10\left(q^{1}\right)^{3}+4 q^{3} \\
& q_{, t t}^{3}=-20 q^{1} q^{3}-8\left(q^{2}\right)^{2}+30\left(q^{1}\right)^{2} q^{2}-15\left(q^{1}\right)^{4}+c
\end{aligned}
$$

(where the subscript, $t$ denotes the differentiation with respect to the evolution parameter $t \in \mathbb{R}$ ). The above system can be represented as a quasi-biHamiltonian system on $\mathcal{N}$ belonging to a quasi-bi-Hamiltonian chain of the form (20) with $n=3$, with Hamiltonians

$$
\begin{aligned}
H_{1} & =p_{1} p_{3}+\frac{1}{2} p_{2}^{2}+10\left(q^{1}\right)^{2} q^{3}-4 q^{2} q^{3}+8 q^{1}\left(q^{2}\right)^{2}-10\left(q^{1}\right)^{3} q^{2}+3\left(q^{1}\right)^{5} \\
H_{2} & =\frac{1}{2} q^{3} p_{3}^{2}-\frac{1}{2} q^{1} p_{2}^{2}+\frac{1}{2} q^{2} p_{2} p_{3}-\frac{1}{2} p_{1} p_{2}-\frac{1}{2} q^{1} p_{1} p_{3}+2\left(q^{1}\right)^{2}\left(q^{2}\right)^{2}+\frac{5}{2}\left(q^{1}\right)^{4} q^{2} \\
& -\frac{5}{4}\left(q^{1}\right)^{6}-2\left(q^{2}\right)^{3}+\left(q^{3}\right)^{2}-6 q^{1} q^{2} q^{3} \\
H_{3} & =\frac{1}{8}\left(q^{2}\right)^{2} p_{3}^{2}+\frac{1}{8}\left(q^{1}\right)^{2} p_{2}^{2}+\frac{1}{8} p_{1}^{2}+\frac{1}{4} q^{1} p_{1} p_{2}+\frac{1}{4} q^{2} p_{1} p_{3}-\frac{1}{4} q^{1} q^{2} p_{2} p_{3} \\
& -\frac{1}{2} q^{3} p_{2} p_{3}-3\left(q^{1}\right)^{3}\left(q^{2}\right)^{2}+q^{1}\left(q^{2}\right)^{3}+\frac{5}{4}\left(q^{1}\right)^{5} q^{2}+2 q^{1}\left(q^{3}\right)^{2} \\
& +\frac{5}{4}\left(q^{1}\right)^{4} q^{3}+\left(q^{2}\right)^{2} q^{3}-\left(q^{1}\right)^{2} q^{2} q^{3}
\end{aligned}
$$

with the corresponding canonical operator $\theta_{0}$ (11) and $\theta_{1}$ of the form

$$
\theta_{1}=\frac{1}{2}\left[\begin{array}{cccccc}
0 & 0 & 0 & q^{1} & -1 & 0 \\
0 & 0 & 0 & q^{2} & 0 & -1 \\
0 & 0 & 0 & 2 q^{3} & q^{2} & q^{1} \\
-q^{1} & -q^{2} & -2 q^{3} & 0 & p_{2} & p_{3} \\
1 & 0 & -q^{2} & -p_{2} & 0 & 0 \\
0 & 1 & -q^{1} & -p_{3} & 0 & 0
\end{array}\right]
$$

and with $\rho_{1}=-q^{1}, \rho_{2}=\frac{1}{4}\left(q^{1}\right)^{2}+\frac{1}{2} q^{2}, \rho_{3}=-\frac{1}{4} q^{1} q^{2}-\frac{1}{4} q^{3}$.

From the form of $H_{1}$ one can directly see that the inverse metric tensor $G$ expressed in $(q, p)$ variables has in this example an anti-diagonal form

$$
G=\frac{1}{2}\left[\begin{array}{lll}
0 & 0 & 1 \\
0 & 1 & 0 \\
1 & 0 & 0
\end{array}\right]
$$

while the conformal Killing tensor $L$ has the form

$$
L=\frac{1}{2}\left[\begin{array}{ccc}
q^{1} & -1 & 0 \\
q^{2} & 0 & -1 \\
2 q^{3} & q^{2} & q^{1}
\end{array}\right]
$$

which substituted in (14) yields the geodesic parts of all the Hamiltonians (40). Our quasi-bi-Hamiltonian system turns out to be separable in $(\lambda, \mu)$ coordinates 
defined as above and it turns out that in this case $f_{i}=1 / 8=$ const and $\gamma_{i}\left(\lambda^{i}\right)=16\left(\lambda^{i}\right)^{7}$ in (21). Thus, we can easily find out the constraints $\rho_{3}=$ $v_{3}=0$ directly in $(q, p)$ coordinates. From the form of $H_{3}$ in (40) one can see that $\varphi_{1}^{\prime \prime}=\rho_{3}(q)=-\frac{1}{4}\left(q_{1} q_{2}+q_{3}\right)$ and that $\varphi_{2}^{\prime \prime}=v_{3}(q, p)$ is just the $g$ consequence of $\rho_{3}$. An easy computation of (23) with the use of (41) yields that $v_{3}(q, p)=-\frac{1}{8}\left(p_{1}+p_{2} q^{1}+p_{3} q^{2}\right)$. Performing the Dirac deformation (8) of $\theta_{0}$ and $\theta_{1}$ with the use of constraints $\varphi_{1}^{\prime \prime}$ and $\varphi_{2}^{\prime \prime}$ yields

$$
\theta_{0, D}=\frac{1}{2 q^{2}+\left(q^{1}\right)^{2}}\left[\begin{array}{ccc|ccc}
0 & 0 & 0 & q^{2}+\left(q^{1}\right)^{2} & -q^{1} & -1 \\
0 & 0 & 0 & -q^{1} q^{2} & 2 q^{2} & -q^{1} \\
0 & 0 & 0 & -\left(q^{2}\right)^{2} & -q^{1} q^{2} & q^{2}+\left(q^{1}\right)^{2} \\
\hline & -*^{T} & q^{2} p_{3}-q^{1} p_{2} & q^{1} p_{2}-q^{2} p_{3} & p_{2} \\
& & -p_{2} & 0 & p_{3} \\
& & & -p_{3} & 0
\end{array}\right]
$$

and

$$
\theta_{1, D}=\frac{1}{2}\left[\begin{array}{cccccc}
0 & 0 & 0 & q^{1} & -1 & 0 \\
0 & 0 & 0 & q^{2} & 0 & -1 \\
0 & 0 & 0 & -2 q^{1} q^{2} & q^{2} & q^{1} \\
-q^{1} & -q^{2} & 2 q^{1} q^{2} & 0 & p_{2} & p_{3} \\
1 & 0 & -q^{2} & -p_{2} & 0 & 0 \\
0 & 1 & -q^{1} & -p_{3} & 0 & 0
\end{array}\right]
$$

so that $\theta_{1, D}$ differs from $\theta_{1}$ only at entries $(3,4)$ and $(4,3)$, where $q^{3}$ was deformed to $-q^{1} q^{2}$ (notice that on $\mathcal{S}$ indeed we have $q^{3}=-q^{1} q^{2}$ ). We will now pass to the Casimir variables chosen as variables

$$
\left(q^{1}, q^{2}, \varphi_{1}^{\prime \prime}(q), \varphi_{2}^{\prime \prime}(q, p), p_{2}, p_{3}\right)
$$

since due to the fact that it is easiest to eliminate $q^{3}$ and $p_{1}$ from the system of equations $\varphi_{1}^{\prime \prime}=\varphi_{1}^{\prime \prime}(q), \varphi_{2}^{\prime \prime}=\varphi_{2}^{\prime \prime}(q, p)$ we will parametrize our submanifold by the coordinates $\left(q^{1}, q^{2}, p_{2}, p_{3}\right)$. In the variables (42) the operators $\theta_{i, D}$ attain the form

$$
\theta_{0, D}=\frac{1}{2 q^{2}+\left(q^{1}\right)^{2}}\left[\begin{array}{cccccc}
0 & 0 & 0 & 0 & -q^{1} & -1 \\
0 & 0 & 0 & 0 & 2 q^{2} & -q^{1} \\
0 & 0 & 0 & 0 & 0 & 0 \\
0 & 0 & 0 & 0 & 0 & 0 \\
q^{1} & 2 q^{2} & 0 & 0 & 0 & p_{3} \\
1 & q^{1} & 0 & 0 & -p_{3} & 0
\end{array}\right]
$$

and

$$
\theta_{1, D}=\frac{1}{2}\left[\begin{array}{cccccc}
0 & 0 & 0 & 0 & -1 & 0 \\
0 & 0 & 0 & 0 & 0 & -1 \\
0 & 0 & 0 & 0 & 0 & 0 \\
0 & 0 & 0 & 0 & 0 & 0 \\
1 & 0 & 0 & 0 & 0 & 0 \\
0 & 1 & 0 & 0 & 0 & 0
\end{array}\right]
$$

respectively. Observe that in these new variables all the entries at third and fourth rows and columns are zero, as it should be, since now the Casimirs $\varphi_{1}^{\prime \prime}$ 
and $\varphi_{2}^{\prime \prime}$ are part of our coordinate system. We can now write down the reduced operators $\theta_{0, R}$ and $\theta_{1, R}$ in variables $\left(q^{1}, q^{2}, p_{2}, p_{3}\right)$ by simply removing the zero rows and columns (we would also have to put $\varphi_{i}^{\prime \prime}=0$ but our matrices $\theta_{i, D}$ do not contain any variables $\varphi_{i}^{\prime \prime}$ in their entries). This yields

$$
\theta_{0, R}=\frac{1}{2 q^{2}+\left(q^{1}\right)^{2}}\left[\begin{array}{cccc}
0 & 0 & -q^{1} & -1 \\
0 & 0 & 2 q^{2} & -q^{1} \\
q^{1} & -2 q^{2} & 0 & p_{3} \\
1 & q^{1} & -p_{3} & 0
\end{array}\right] \quad, \theta_{1, R}=-\frac{1}{2}\left[\begin{array}{cc}
0_{2} & I_{2} \\
-I_{2} & 0_{2}
\end{array}\right]
$$

so that $\theta_{1, R}$ attains in the variables $\left(q^{1}, q^{2}, p_{2}, p_{3}\right)$ the canonical form. An easy calculation yields that the Hamiltonians $H_{i}$ restricted to $\mathcal{S}$ become

$$
\begin{aligned}
H_{1, R}\left(q^{1}, q^{2}, p_{2}, p_{3}\right) & =\left.H_{1}\right|_{\mathcal{S}}=-q^{1} p_{2} p_{3}+\frac{1}{2} p_{2}^{2}-q^{2} p_{3}^{2}-20\left(q^{1}\right)^{3} q^{2} \\
& +12 q^{1}\left(q^{2}\right)^{2}+3\left(q^{1}\right)^{5} \\
H_{2, R}\left(q^{1}, q^{2}, p_{2}, p_{3}\right) & =\left.H_{2}\right|_{\mathcal{S}}=\left(q^{2}+\frac{1}{2}\left(q^{1}\right)^{2}\right) p_{2} p_{3}+9\left(q^{1}\right)^{2}\left(q^{2}\right)^{2} \\
& +\frac{5}{2}\left(q^{1}\right)^{4} q^{2}-\frac{5}{4}\left(q^{1}\right)^{6}-2\left(q^{2}\right)^{3} \\
H_{3, R}\left(q^{1}, q^{2}, p_{2}, p_{3}\right) & =\left.H_{3}\right|_{\mathcal{S}}=0
\end{aligned}
$$

while the functions $\rho_{i, R}$ are: $\rho_{1, R}=-q^{1}, \rho_{2, R}=\frac{1}{4}\left(q^{1}\right)^{2}+\frac{1}{2} q^{2}, \rho_{3, R}=0$. Thus, we have obtained on $S$ a new separable quasi-bi-Hamiltonian system of the form (20) with $n=2$. This concludes our first example.

As a second example we will consider a separable (quasi)-bi-Hamiltonian system with a non-trivial function $f$. More specifically, we shall consider a Lagrangian system of second order Newton equations

$$
\begin{aligned}
& q_{, t t}^{1}=16 q^{1}\left(1+q^{2}\right) \\
& q_{, t t}^{2}=8\left(q^{1}\right)^{2}+4\left(q^{3}\right)^{2}+64 q^{2}+48\left(q^{2}\right)^{2}+4 c+24 \\
& q_{, t t}^{3}=4 q^{3}\left(3+4 q^{2}\right)
\end{aligned}
$$

This system is a part of a quasi-bi-Hamiltonian chain with Hamiltonians

$$
\begin{aligned}
H_{1} & =p_{1}^{2}+p_{2}^{2}+p_{3}^{2}-4\left(q^{1}\right)^{2}\left(1+q^{2}\right)-\left(q^{3}\right)^{2}\left(3+4 q^{2}\right) \\
& -4\left(3 q^{2}+4\left(q^{2}\right)^{2}+2\left(q^{2}\right)^{3}\right) \\
H_{2} & =-\left(2 q^{2}+1\right) p_{1}^{2}+2 q^{1} p_{1} p_{2}-p_{2}^{2}+2 q^{3} p_{2} p_{3}-2 p_{3}^{2}\left(q^{2}+1\right) \\
& -\left(q^{1}\right)^{2}\left(\left(q^{1}\right)^{2}+2\left(q^{3}\right)^{2}+4\left(q^{2}\right)^{2}+4 q^{2}+2\right)-\left(q^{3}\right)^{2}\left(\left(q^{3}\right)^{2}+4\left(q^{2}\right)^{2}+2 q^{2}\right) \\
& +8\left(q^{2}\right)^{3}+16\left(q^{2}\right)^{2}+12 q^{2} \\
H_{3} & =-\left(q^{3}\right)^{2} p_{1}^{2}+2 q^{1} q^{3} p_{1} p_{3}-2 q^{3} p_{2} p_{3}+\left(1+2 q^{2}-\left(q^{1}\right)^{2}\right) p_{3}^{2} \\
& +\left(\left(q^{1}\right)^{2}+4\left(q^{2}\right)^{2}+\left(q^{3}\right)^{2}+6 q^{2}+3\right)\left(q^{3}\right)^{2},
\end{aligned}
$$


with the canonical Poisson tensors $\theta_{0}$ and

$$
\theta_{1}=\left[\begin{array}{cccccc}
0 & 0 & 0 & 1 & q^{1} & 0 \\
0 & 0 & 0 & q^{1} & 2 q^{2}+1 & q^{3} \\
0 & 0 & 0 & 0 & q^{3} & 0 \\
-1 & -q^{1} & 0 & 0 & -p_{1} & 0 \\
-q^{1} & -2 q^{2}-1 & -q^{3} & p_{1} & 0 & p_{3} \\
0 & -q^{3} & 0 & 0 & -p_{3} & 0
\end{array}\right]
$$

and with the functions

$$
\rho_{1}(q)=-2\left(q^{2}+1\right), \rho_{2}(q)=1-\left(q^{3}\right)^{2}-\left(q^{1}\right)^{2}+2 q^{2}, \rho_{3}(q)=\left(q^{3}\right)^{2}
$$

The above system is a particular example of so called bi-cofactor systems [27], [28, 23], 29], 30] separated recently in [15]. In Darboux-Nijenhuis coordinates $(\lambda, \mu)$ it turns out that now $f\left(\lambda^{i}\right)=4 \lambda^{i}\left(\lambda^{i}-1\right)$ and $\gamma_{i}\left(\lambda^{i}\right)=\left(\lambda^{i}\right)^{5}$. This means that in order to find the constraint $\varphi_{2}^{\prime \prime}(q, p)$ we have to use the formula (36) with $\varphi_{1}^{\prime \prime}(q)=\left(q^{3}\right)^{2}$. Plugging these functions into (36) and using the above forms of $G$ and $F$ we get a rather complicated expression for $\varphi_{2}^{\prime \prime}$

$$
\varphi_{2}^{\prime \prime}(q, p) \equiv v_{3}(q, p)=\frac{1}{4}\left(p_{1} \frac{\left(q^{1}\right)^{2}+\left(q^{3}\right)^{2}}{q^{1}}-p_{2}+p_{3}\left(-q^{3}+\frac{2 q^{2}+\left(q^{1}\right)^{2}-1}{q^{3}}\right)\right)
$$

Notice that the constraint $\varphi_{2}^{\prime \prime}$ seems to have a singularity on the surface $\varphi_{1}^{\prime \prime}=0$. However, it turns out that on the submanifold $\mathcal{S}=\left\{\varphi_{1}=\varphi_{2}=0\right\}$ we have $p_{3}=$ 0 and the singularity disappears (see below). Having obtained the constraints $\varphi_{1}^{\prime \prime}$ and $\varphi_{2}^{\prime \prime}$ in $(q, p)$ variables we now proceed as in the first example. First we find

$$
\theta_{0, D}=\left[\begin{array}{cccccc}
0 & 0 & 0 & 1 & 0 & \zeta_{1} \\
0 & 0 & 0 & 0 & 1 & \zeta_{2} \\
0 & 0 & 0 & 0 & 0 & 0 \\
-1 & 0 & 0 & 0 & 0 & \zeta_{3} \\
0 & -1 & 0 & 0 & 0 & \zeta_{4} \\
-\zeta_{1} & -\zeta_{2} & 0 & -\zeta_{3} & -\zeta_{4} & 0
\end{array}\right]
$$

(where $\zeta_{i}$ are some rational functions of $q$ and $p$ that do not vanish on $\mathcal{S}$ ) and

$$
\theta_{1, D}=\left[\begin{array}{cccccc}
0 & 0 & 0 & 1 & \frac{\left(3^{3}\right)^{2}}{q^{1}}+q^{1} & 0 \\
0 & 0 & 0 & q^{1} & 2 q^{2}+1 & q^{3} \\
0 & 0 & 0 & 0 & 0 & 0 \\
-1 & -q^{1} & 0 & 0 & p_{1}\left(\frac{\left(q^{3}\right)^{2}}{\left(q^{1}\right)^{2}}-1\right) & 0 \\
-\frac{\left(q^{3}\right)^{2}}{q^{1}}-q^{1} & -2 q^{2}-1 & 0 & p_{1}\left(1-\frac{\left(q^{3}\right)^{2}}{\left(q^{1}\right)^{2}}\right) & 0 & 2 p_{3} \\
0 & -q^{3} & 0 & 0 & -2 p_{3} & 0
\end{array}\right]
$$

(notice that in both cases $q^{3}$ is a Casimir function). It can be checked that the Schouten bracket of $\theta_{0, D}$ and $\theta_{1, D}$ is equal to zero: $\left[\theta_{0, D}, \theta_{1, D}\right]_{S}=0$ so that 
the operators $\theta_{0, D}$ and $\theta_{1, D}$ are compatible. Before projecting $\theta_{0, D}$ and $\theta_{1, D}$ onto $\mathcal{S}$ we perform a transformation of variables to Casimir variables

$$
\left(q^{1}, q^{2}, \varphi_{1}^{\prime \prime}(q), p_{1}, p_{2}, \varphi_{2}^{\prime \prime}(q, p)\right)
$$

since this time it is easier to eliminate $q^{3}$ and $p_{3}$ from the system of equations $\varphi_{1}^{\prime \prime}=\varphi_{1}^{\prime \prime}(q), \varphi_{2}^{\prime \prime}=\varphi_{2}^{\prime \prime}(q, p)$. Explicitly we have

$$
\begin{aligned}
& q^{3}= \pm \sqrt{\varphi_{1}^{\prime \prime}} \\
& p_{3}=\sqrt{\varphi_{1}^{\prime \prime}} \frac{4 \varphi_{2}^{\prime \prime} q^{1}+\varphi_{1}^{\prime \prime} p_{1}+\left(q^{1}\right)^{2} p_{1}-q^{1} p_{2}}{2 q^{1} q^{2}+q^{1}-q^{1} \varphi_{1}^{\prime \prime}-\left(q^{1}\right)^{3}}
\end{aligned}
$$

As we have mentioned, we have now $\left.p_{3}\right|_{\mathcal{S}}=0$. We will thus parametrize our submanifold $\mathcal{S}$ by the coordinates $\left(q^{1}, q^{2}, p_{1}, p_{2}\right)$. After expressing the operators $\theta_{i, D}$ in the variables (44) with the help of relations (45) we can easily project these operators on $\mathcal{S}$. As the result we get a new (reduced) quasi-bi-Hamiltonian chain of the form (201) with $n=2$, which is the variables $\left(q^{1}, q^{2}, p_{1}, p_{2}\right)$ is determined by

$$
\theta_{0, R}=\left[\begin{array}{ll}
0_{2} & I_{2} \\
I_{2} & 0_{2}
\end{array}\right] \quad, \quad \theta_{1, R}=\left[\begin{array}{cccc}
0 & 0 & 1 & q^{1} \\
0 & 0 & q^{1} & 2 q^{2}+1 \\
-1 & -q^{1} & 0 & -p_{1} \\
-q^{1} & -2 q^{2}-1 & p_{1} & 0
\end{array}\right]
$$

and by the restricted Hamiltonians $H_{i}$

$$
\begin{gathered}
H_{1, R}=\left.H_{1}\right|_{\mathcal{S}}=p_{1}^{2}+p_{2}^{2}-4\left(q^{1}\right)^{2}\left(1+q^{2}\right)-4\left(3 q^{2}+4\left(q^{2}\right)^{2}+2\left(q^{2}\right)^{3}\right) \\
\begin{aligned}
H_{2, R}= & \left.H_{2}\right|_{\mathcal{S}}=-\left(1+2 q^{2}\right) p_{1}^{2}+2 q^{1} p_{1} p_{2} q-p_{2}^{2} \\
& \quad-\left(q^{1}\right)^{2}\left(\left(q^{1}\right)^{2}+2\left(q^{3}\right)^{2}+4\left(q^{2}\right)^{2}+4 q^{2}+2\right) \\
\quad & 8\left(q^{2}\right)^{3}+16\left(q^{2}\right)^{2}+12 q^{2} \\
H_{3, R}= & \left.H_{3}\right|_{\mathcal{S}}=0
\end{aligned}
\end{gathered}
$$

while the functions $\rho_{i, R}$ are given by $\rho_{1, R}=-2 c\left(q^{2}+1\right), \rho_{2, R}=1+2 q^{2}-\left(q^{1}\right)^{2}$, $\rho_{3, R}=0$.

Notice that when we take $\varphi_{2}^{\prime \prime}(q, p)=2 q^{3} p_{3}$, i.e. the differential consequence of $\varphi_{1}^{\prime \prime}(q, p)=\left(q^{3}\right)^{2}$, then $\left\{\varphi_{1}^{\prime \prime}, \varphi_{2}^{\prime \prime}\right\}_{\theta_{1}}=0$ and the Dirac deformation $\theta_{1, D}$ does not exists (see Remark 10).

\section{Conclusions}

In this article we have focused on the problem of Dirac deformation and Dirac reduction of Poisson operators and Poisson pencils. We have presented a procedure of performing Dirac reduction in quasi-bi-Hamiltonian systems of Benenti type that do not destroy the separability of these systems and that moreover do not change the separation variables. The method presented is not, however, 
general in the sense that it does not provide us with all such reductions, but only with their subclass. Moreover, for the moment the procedure works only for those systems, for which the functions $f_{i}$ are all equal, i.e. when $f_{i}$ does not depend on $i$. Last but not least, the presented procedure is valid only in case when one of the Poisson structures of the system is canonical - the non-canonical

case must be studied separately. These issues will be addressed in a separate paper.

\section{References}

[1] C. Morosi, G. Tondo, "Quasi-bi-Hamiltonian systems and separability", $J$. Phys. A: Math. Gen. 30, (1997), 2799.

[2] Błaszak M, "On separability of bi-Hamiltonian chain with degenerated Poisson structures", J. Math. Phys. 39, 3213 (1998)

[3] Błaszak M, "Bi-Hamiltonian separable chains on Riemannian manifolds", Phys. Lett. A 243, 25 (1998)

[4] Błaszak M, "Theory of separability of multi-Hamiltonian chains", J. Math. Phys. 40 (1999) 5725.

[5] Błaszak M, "Inverse bi-Hamiltonian separable chains", J. Theor. Math. Phys. 122 (2000) 140.

[6] Błaszak M, "Separability of two-Casimir bi- and tri-Hamiltonian chains", Rep. Math. Phys. 46 (2000) 35.

[7] Błaszak M, "Degenerate Poisson Pencils on Curves: New Separability Theory", J. Nonl. Math.Phys. 7 (2000). 213

[8] Falqui G, Magri F. and Tondo G, "Reduction of bihamiltonian systems and separation of variables: an example from the Boussinesq hierarchy", Theor. Math. Phys. 122 (2000) 176.

[9] Falqui G, Magri F, Pedroni M, "Bihamiltonian geometry and separation of variables for Toda lattices", eprint nlin.SI/0002008 (2000).

[10] Błaszak M, "From bi-Hamiltonian geometry to separation of variables: stationary Harry-Dym and the KdV dressing chain", Journal of Nonlinear Mathematical Physics, 9 Supplement 1 (2002) 1

[11] Ibort A, Magri F., Marmo G, "Bihamiltonian structures and Stäckel separability", Journal of Geometry and Physics 33 (2000) 210-228

[12] Falqui G., Magri F., Pedroni M., Zubelli J.P., "A Bi-Hamiltonian Theory for Stationary KdV flows and their Separability", Regular Chaotic Dynamics 5 (2000) 33 
[13] Falqui G, Magri F, Pedroni M, "Bihamiltonian geometry and separation of variables for Toda lattices", eprint nlin.SI/0002008 (2000)

[14] Falqui G, Magri F. and Tondo G, "Reduction of bihamiltonian systems and separation of variables: an example from the Boussinesq hierarchy", Theor. Math. Phys. 122 (2000) 176

[15] K. Marciniak, M. Blaszak, "Separation of variables in quasi-potential systems", J. Phys. A: Math. Gen. 35 (2001) 2947

[16] P. A. M. Dirac, "Generalized Hamiltonian Dynamics", Can. J. Math. 2 (1950) 129-148

[17] P. A. M. Dirac, Lecture Notes on Quantum Mechanics, Yeshova University, New York, 1964

[18] F. Magri, "A simple model of the integrable Hamiltonian equation", $J$. Math. Phys. 19 (5), 1978, pp. 1156-1162

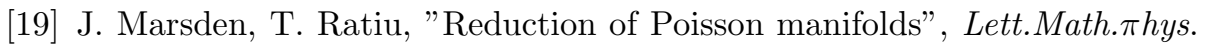
11 (1986) 161-169

[20] G. Falqui, M. Pedroni, "On Poisson reduction for Gel'fand-Zakharevich manifolds", to appear in Rep. Math. Phys.

[21] S. Benenti, "Orthogonal separable dynamical systems", in: Differential geometry and its applications, Proceedings on the Conference in Opava, 2428 August 1992, Silesian University, Opava, vol. I, 1993, p. 163; Electronic edition http:/www.emis.de/proceedings/

[22] S. Benenti, "Intrinsic characterization of the variable separation in the Hamilton-Jacobi equation", J. Math. Phys. 39 (1997) 6578-6602

[23] H. Lundmark, "A new class of integrable Newton systems", Journal of Nonlinear Mathematical Physics, 8, (2001) 195-199, Supplement - Proceedings of NEEDS '99, Kolymbari, Crete.

[24] P. Vanhaecke, "Integrable systems in the Realm of Algebraic Geometry", Lecture Notes in Mathematics 1638, Springer-Verlag, New York, 1996

[25] F. Magri and T. Marsico, "Some Developments of the Concept of Poisson Manifolds in the Sense of A. Lichnerowicz", in Gravitation, Electromagnetism and Geometric Structures, ed. G. Ferrarese, Pitagora editrice, Bologna, 1996, p.207

[26] S. Rauch-Wojciechowski, K. Marciniak, M. Blaszak, "Two Newton decompositions of stationary flows of KdV and Harry Dym hierarchies" Physica A 233 (1996) pp.307-330 
[27] Marciniak K, Rauch-Wojciechowski S, "Two families of nonstandard Poisson structures for Newton equations", J. Math. Phys. 39 (10), 1998, 52925306

[28] Rauch-Wojciechowski S, Marciniak K, Lundmark H, "Quasi-Lagrangian systems of Newton equations", J. Math. Phys. 40 (12), 1999, 6366-6398.

[29] H. Lundmark, "Higher-dimensional integrable Newton systems with quadratic integrals of motion", to appear in Stud.Appl.Math.

[30] M. Crampin, W. Sarlet, "A class of non-conservative Lagrangian systems on Riemannian manifolds", J. Math. Phys. 42 (9), 2001, pp. 4313-4326. 